\title{
Cluster formation for multi-strain infections with cross-immunity
}

\author{
V. Calvez ${ }^{\mathrm{a}, 1}$, A. Korobeinikov ${ }^{\mathrm{b}, *, 2}$, P.K. Maini ${ }^{\mathrm{b}, 3}$

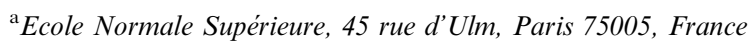 \\ ${ }^{\mathrm{b}}$ Centre for Mathematical Biology, Mathematical Institute, University of Oxford, 24-29 St Giles', Oxford OXI 3LB, UK
}

Received 7 October 2003; received in revised form 21 September 2004; accepted 23 September 2004

Available online 30 November 2004

\begin{abstract}
Many infectious diseases exist in several pathogenic variants, or strains, which interact via cross-immunity. It is observed that strains tend to self-organise into groups, or clusters. The aim of this paper is to investigate cluster formation. Computations demonstrate that clustering is independent of the model used, and is an intrinsic feature of the strain system itself. We observe that an ordered strain system, if it is sufficiently complex, admits several cluster structures of different types. Appearance of a particular cluster structure depends on levels of cross-immunity and, in some cases, on initial conditions. Clusters, once formed, are stable, and behave remarkably regularly (in contrast to the generally chaotic behaviour of the strains themselves). In general, clustering is a type of self-organisation having many features in common with pattern formation.
\end{abstract}

(C) 2004 Elsevier Ltd. All rights reserved.

MSC: primary 92D30; 34D20

Keywords: Mathematical modelling; Infectious disease; Cross-immunity; Self-organisation; Cluster

\section{Introduction}

Many pathogens have several different antigenic variants, or strains, present in a host population simultaneously. The classic example is influenza (Andreasen et al., 1997; Lin et al., 1999; Plotkin et al., 2002; Gog and Grenfell, 2002; Cliff et al., 1986), where there are several circulating subtypes, with many minor variants within each subtype. Other important examples

\footnotetext{
*Corresponding author. Current address: RIES, University of Hokkaido, N12W6 Sapporo, 060-0812, Japan. Tel.: + 81117062891 ; fax: +81117064966 .

E-mail addresses: vincent.calvez@ens.fr (V. Calvez), andrei@nsc. es.hokudai.ac.jp (A. Korobeinikov), maini@maths.ox.ac.uk (P.K. Maini).

${ }^{1}$ VC thank Dr. S. Gupta and Mr. M. Recker for helpful discussions and for providing a preprint.

${ }^{2} \mathrm{AK}$ is supported by the Foundation for Research, Science and Technology of New Zealand, through Project Contract UOXX0101.

${ }^{3}$ PKM was supported in part by National Science Foundation under Grant No. PHY99-07949. PKM would like to thank KITP, UC Santa Barbara for their kind hospitality.
}

are meningitis (Gupta et al., 1996; Gupta and Anderson, 1999), dengue (Gog and Grenfell, 2002) and malaria (Gupta et al., 1994).

Because of similarities in, for example, their mechanisms of infection, strains may interact with each other (Gupta et al., 1996). Infection with one strain may partially protect the host against infection with other strains. Cross-immunity is included in different ways in different models, but the general idea is the same: infection with one strain of the disease produces a lasting immune memory in the host which acts to protect against subsequent infection by other strains. That is, for two sufficiently close strains $A$ and $B$, infection by strain $A$ reduces the chance of a secondary infection by strain $B$. For instance, in the case of influenza, the surface protein hemagglutinin seems to be under strong positive selection because it is the target of the immune response, and therefore it presents high antigenic diversity in the virus population (Andreasen et al., 1997; Lin et al., 1999; Plotkin et al., 2002; Gog and Grenfell, 2002). This immune response may be 
enhanced because of a previous infection with a close variant.

There are different approaches to the cross-immunity problem (Gog and Swinton, 2002). For instance, we can assume that a fraction, say $\gamma_{B A}$, of individuals infected with strain $A$ gain complete immunity to strain $B$; alternatively, all the individuals infected with strain $A$ may be assumed to acquire partial immunity against $B$ (with a consequence that the force of secondary $B$ infection is reduced by a factor $\gamma_{B A}$ ). Another possible hypothesis is that the secondary infection is weaker and thus less transmissible by the infective host. These differences in the approaches to cross-immunity lead to a variety of models which can provide controversial outcomes. Under such circumstances it is reasonable to look for such features of the multi-strain system which are intrinsic to this system and are robust irrespective of model choice.

A system of multiple strains interacting via host crossimmunity tends to self-organise into groups, or clusters. The tendency for strains to occur in clusters reflects the observed influenza dynamics (Gog and Grenfell, 2002; Plotkin et al., 2002). Cluster formation was observed and discussed by Gupta et al. (1996, 1998). The phenomenon of clustering appears to be typical for many systems with internal order and may occur in such systems as multi-species predator-prey systems. For example, it was observed in neuronal networks (Rubin and Terman, 2000a,b; Terman and Lee, 1997; Terman et al., 1998).

In this paper we consider formation of clusters in ordered multi-strain systems. We show that for complex systems several different types of cluster structure may arise. We also demonstrate that cluster structures are not specific to a particular model —on the contrary, they appear to be intrinsic to the given strain system. In general, cluster formation is a self-organisation phenomenon bearing many similarities to pattern formation. A remarkable feature of clusters is that they exhibit exceptional regularity even when the dynamics of every strain is chaotic.

\section{Model}

Due to different approaches to cross-immunity, a variety of models of multi-strain infections has been developed. These models sometimes lead to different outcomes. It is important, therefore, to find such indicators which are characteristic to the system itself and robust to choice of model.

We start from a comparatively simple model of a multi-strain infection suggested by Gupta et al. (1998). This model is composed of only three compartments (and, respectively, three differential equations) for each strain. If $z_{i}(t)$ is the fraction of individuals who have been or are infected with the strain $i$ (either they are infectious or not), $y_{i}(t)$ is the fraction of the infectious individuals with the strain, and $w_{i}(t)$ is the fraction of individuals who have been infected (or are infected) by any strain sufficiently close to the strain $i$ including $i$ itself (that is $w_{i}=\cup_{j \sim i} z_{j}$ ), then the model equations are:

$$
\begin{aligned}
\frac{\mathrm{d} z_{i}}{\mathrm{~d} t} & =\beta_{i} y_{i}\left(1-z_{i}\right)-\mu z_{i}, \\
\frac{\mathrm{d} w_{i}}{\mathrm{~d} t} & =\sum_{j \sim i} \beta_{j} y_{j}\left(1-w_{i}\right)-\mu w_{i}, \\
\frac{\mathrm{d} y_{i}}{\mathrm{~d} t} & =\beta_{i} y_{i}\left[\left(1-w_{i}\right)+(1-\gamma)\left(w_{i}-z_{i}\right)\right]-\left(\mu+\sigma_{i}\right) y_{i} .
\end{aligned}
$$

For this model, cross-protection does not affect susceptibility but reduces transmissibility by a factor $1-\gamma$ (where the parameter $\gamma$ measures the degree of cross-protection between two strains). Here, $j \sim i$ means that the $j$ th strain is related to the $i$ th strain and can induce cross-protection (that is if $j \sim i$ then $\gamma_{i j} \neq 0$ ). The parameters $1 / \mu$ and $1 / \sigma$ are, respectively, host life expectancy and average period of infectiousness, $\beta$ is transmission rate. We refer to this model as Gupta's model. This simple model has been analysed in Gupta et al. (1998) and provided important insights into pathogen formation and the genetic organisation of strains.

To study the phenomenon of clustering we need to consider several levels of cross-protection. Whereas the original model implies only one level of cross-protection ( $\gamma$ if two strains are related, or zero if they are not) and neglects possible multiple infections by strains related to $i$. We relax these assumptions below to make the model more generally applicable, while striving to keep the model simple. We assume that the probability of cross-protection between strains $i$ and $j$ is $\gamma_{i j}$ (that is, infection by the strain $j$ reduces the probability that the host will be infected by the strain $i$ by a factor $\gamma_{i j}$ ), and consider the barycentre of $\gamma_{i j}$, defined as

$\Gamma_{i}=\left(\sum_{j \sim i, j \neq i} \gamma_{i j} \beta_{j} y_{j}\right) /\left(\sum_{j \sim i, j \neq i} \beta_{j} y_{j}\right)$.

We replace the coefficient $\gamma$ in the system (1) with the barycentre $\Gamma_{i}$. Substituting the barycentre $\Gamma_{i}$ into (1) and using the variables $V_{i}=1-z_{i}, X_{i}=1-w_{i}, Y_{i}=$ $\frac{\beta_{i}}{\mu} y_{i}$ and $\tau=\mu t$, we obtain the system

$$
\begin{aligned}
& \frac{\mathrm{d} V_{i}}{\mathrm{~d} \tau}=1-\left(1+Y_{i}\right) V_{i}, \\
& \frac{\mathrm{d} X_{i}}{\mathrm{~d} \tau}=1-\left(1+\sum_{j \sim i} Y_{j}\right) X_{i}, \\
& \varepsilon_{i} \frac{\mathrm{d} Y_{i}}{\mathrm{~d} \tau}=\left(\left(1-\Gamma_{i}\right) V_{i}+\Gamma_{i} X_{i}-r_{i}\right) Y_{i} .
\end{aligned}
$$

Here $\varepsilon_{i}=\mu / \beta_{i}$ and $r_{i}=\left(\mu+\sigma_{i}\right) / \beta_{i}$. Obviously, $\Gamma_{i} \equiv \gamma$ for Gupta's model (when $\gamma_{i j}$ is either $\gamma$, or zero). 
Furthermore, computations show that for this model the function $\Gamma_{i}(t)$ mostly takes one of a few constant values, with rapid shifting between these values (see Fig. 4(b)); this justifies the use of the function $\Gamma_{i}(t)$.

\section{Structure of a strain set}

Systems of strains were formed as a result of a genetic process, and they generally inherited some internal order associated with this process. Having this intrinsic order, a system of strains may be organised in an ordered set, or a discrete strain space every point of which represents a strain. The idea of the strain space allows us to use the concept of "immunological distance". The immunological distance between two strains may be assumed to depend inversely on their mutual level of crossprotection.

The structure of the strain space depends on underlying immunological and genetic processes. For instance, Gog and Grenfell (2002) considered the simplest possible strain space: a linear strain space. In this case strains are arranged in a line, and they postulated $\gamma_{i j}=$ $\exp \left(-\left(\frac{i-j}{d}\right)^{2}\right)$, where $d$ is a constant. A multi-dimensional strain space may be organised in the same way, with immunological distance defined, for example, as the sum of horizontal and vertical distances. Dawes and Gog (2002) and Gog and Swinton (2002) considered a system of four strains arranged in a circle. In this case, each strain is assumed to interact more strongly with its adjacent neighbours than with the strain opposite.

Studying the maintenance of strain structure in a recombining virus population, Gupta et al. (1996) have introduced a simple framework where strains are organised as follows: each strain is characterised by a combination of alleles at loci which are of immunological interest. Strains induce cross-immunity if they share at least one allele. For example, in the case of two loci and two possible alleles at each locus (say $a$ or $b$ for

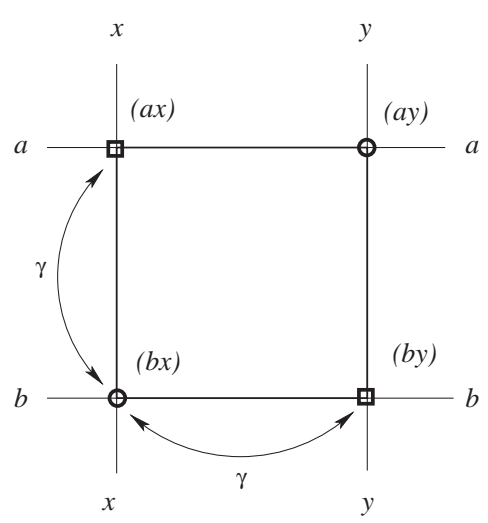

Fig. 1. Strain space of a four-strain system: two loci and two possible alleles at each locus (see text for details).

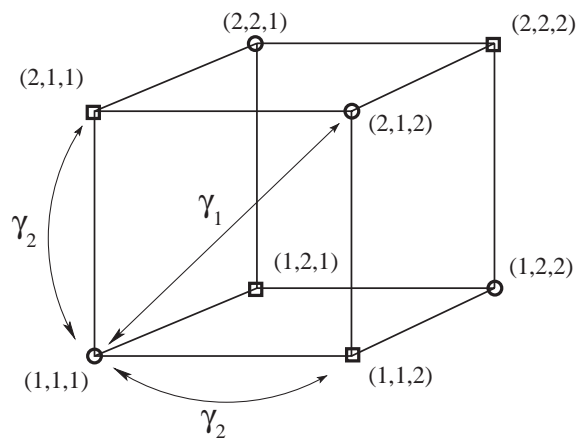

Fig. 2. Strain space of an eight-strain system: three loci with two alleles possible at each locus. Here, for instance, $(2,2,1)$ means that the second, the second and the first alleles are, respectively, at the first, the second and the third locus.

the first locus, and $x$ or $y$ for the second one, respectively) there are four different strains: the original strains $a x$ and $b y$, and the recombinant strains $a y$ and $b x$. To visualise such a strain structure we will use a multi-dimensional graph where a dimension corresponds to a locus, and vertices represent strains. Fig. 1 illustrates the structure of the above mentioned fourstrain system (two loci and two possible alleles at each locus). Fig. 2 shows the strain space of an eight-strain system organised on three loci with two alleles at each locus.

\section{Results}

Cross-immunity may structure a set of strains into groups, or clusters. These groups can behave at least in three ways: remain in homogeneous equilibrium when no structure is observed (Fig. 3a), oscillate when the clusters alternate recurrently in succession (Fig. 3b), or one group may dominate with the others driven below survival level (Fig. 3c) (Gupta et al., 1996, 1998). The phenomenon of clustering is conserved for all sufficiently large levels of cross-protection. Of course, when $\gamma \rightarrow 0$, the equations are decoupled, and the clustering disappears.

In the case of the four-strain system shown in Fig. 1 it is natural to expect the formation of two clusters of nonoverlapping (or discordant) strains, namely $a x$ groups with $b y$, and $a y$ groups with $b x$ (in Fig. 1 we, respectively, mark the strains by squares and circles). Indeed, such clustering has been observed (Gog and Swinton, 2002; Dawes and Gog, 2002; Gupta et al., 1996, 1998). Fig. 3 illustrates the strain dynamics: it is easy to see the formation of two clusters.

However, a multi-strain system with only one level of cross-protection which is the same for all related strains, is hardly realistic. As the number of strains grows, and 

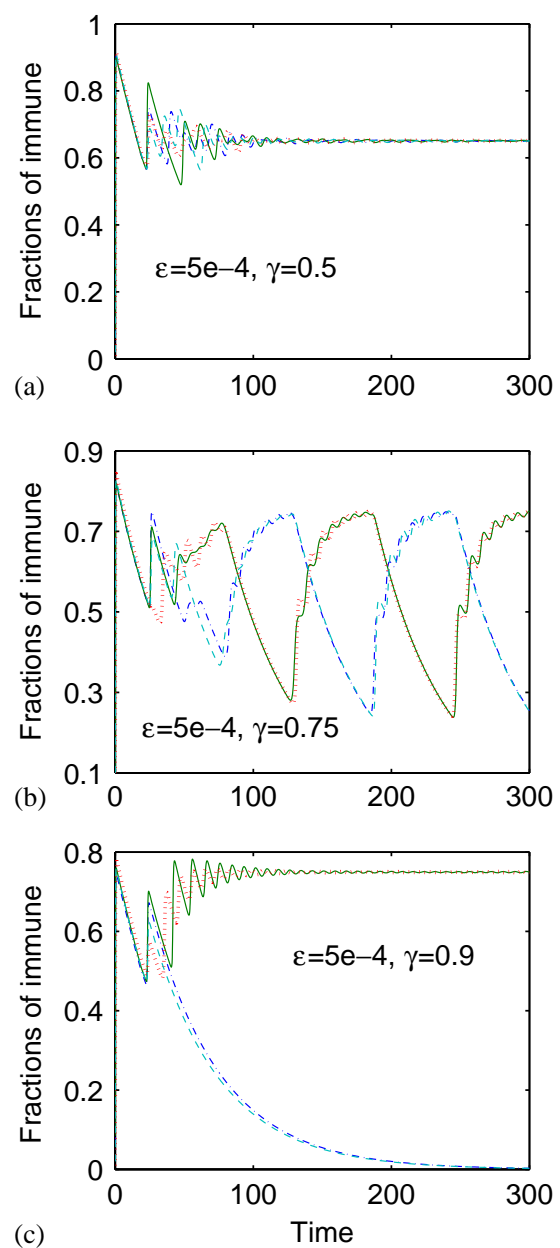

Fig. 3. Dynamics of the four-strain system shown in Fig. 1. Here (b) and (c) illustrate formation of two clusters each consisting of two strains; in (a) the system is in homogeneous equilibrium, and no definite clustering can be observed.

especially if there are several different levels of cross-protection, the self-organisation of the system may be more complicated. Furthermore, it may be different for different levels of cross-protection. For instance, for the eight-strain system shown in Fig. 2 at least two different types of clustering are possible. From now on we will use the terms cluster structure and type of cluster structure. The difference between these objects is that different cluster structures may be of the same type. Below we will show this using an example.

For a system of eight strains organised in three loci with two alleles each (Fig. 2) we assume two levels of cross-protection: namely $\gamma_{1}$ if the strains share one allele, or $\gamma_{2}$ if they share two alleles. Naturally, $\gamma_{1} \leqslant \gamma_{2}$. For this system one can expect formation of a structure of four clusters with two discordant strains each (Gupta et al., 1998). Every cluster of such structure corresponds to one of the four main diagonals of the cube in Fig. 2.
However, this type of cluster structure was observed only when $\gamma_{1}$ and $\gamma_{2}$ are sufficiently close. As the difference between $\gamma_{1}$ and $\gamma_{2}$ grows, a new type of cluster structure appears: now there are two clusters, $\alpha$ and $\beta$, with four strains each ( $\alpha$ is composed of the strains $(1,1,1),(1,2,2),(2,1,2)$ and $(2,2,1)$, and $\beta$ of $(2,2,2)$, $(2,1,1),(1,2,1)$ and $(1,1,2))$. In Fig. 2 the strains of these "tetrahedral" clusters are marked, respectively, by circles and squares. This second type of clustering can hardly be expected a priori. However, this cluster structure exists for a much wider range of $\gamma_{1}$ and $\gamma_{2}$ than the first type. Fig. 4 illustrates the dynamics of the second type of clustering. Here, the logarithm of the force of infection $\log Y_{i}(t)$ (Fig. 4(a)) and the effective cross-protection $\Gamma_{\alpha}(t)$ (Fig. 4(b)) are shown for $\varepsilon=$ $5 \times 10^{-3}$ (left column) and $5 \times 10^{-4}$ (right column). Note that the function $\Gamma_{\alpha}(t)$ remains constant most of the time, with rapid shifting between two constant values. Also note the remarkable regularity of the function $\Gamma_{\alpha}(t)$ in contrast to the chaotic behaviour of the forces of infection. The function $\Gamma_{\beta}(t)$ is qualitatively the same.

Fig. 5 shows the results of stochastic perturbation of the system. Here we assume that $1 / \varepsilon=200+\delta(t)$, where $\delta(t)$ is a Gaussian-distributed noise of magnitude 1 (a), 10 (b) and 20 (c). It is easy to see that the cluster structure is robust to such stochastic perturbations.

A cluster structure may be qualitatively defined by the clustering matrix $M$ : we set $m_{i j}$ equal to 1 if the strains $i$ and $j$ belong to the same cluster, and $m_{i j}=0$ otherwise. Naturally, the matrix is symmetric and $m_{i i}=1$. For instance, if the vertices of the cube in Fig. 2 are ordered as follows:

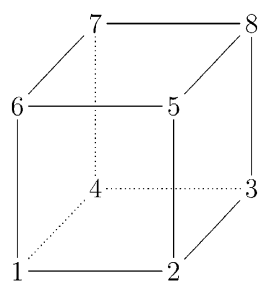

then the clustering matrices for the first and second types of clustering are

$$
M_{I}=\left(\begin{array}{cccccccc}
1 & 0 & 0 & 0 & 0 & 0 & 0 & 1 \\
0 & 1 & 0 & 0 & 0 & 0 & 1 & 0 \\
0 & 0 & 1 & 0 & 0 & 1 & 0 & 0 \\
0 & 0 & 0 & 1 & 1 & 0 & 0 & 0 \\
0 & 0 & 0 & 1 & 1 & 0 & 0 & 0 \\
0 & 0 & 1 & 0 & 0 & 1 & 0 & 0 \\
0 & 1 & 0 & 0 & 0 & 0 & 1 & 0 \\
1 & 0 & 0 & 0 & 0 & 0 & 0 & 1
\end{array}\right) \text { and }
$$



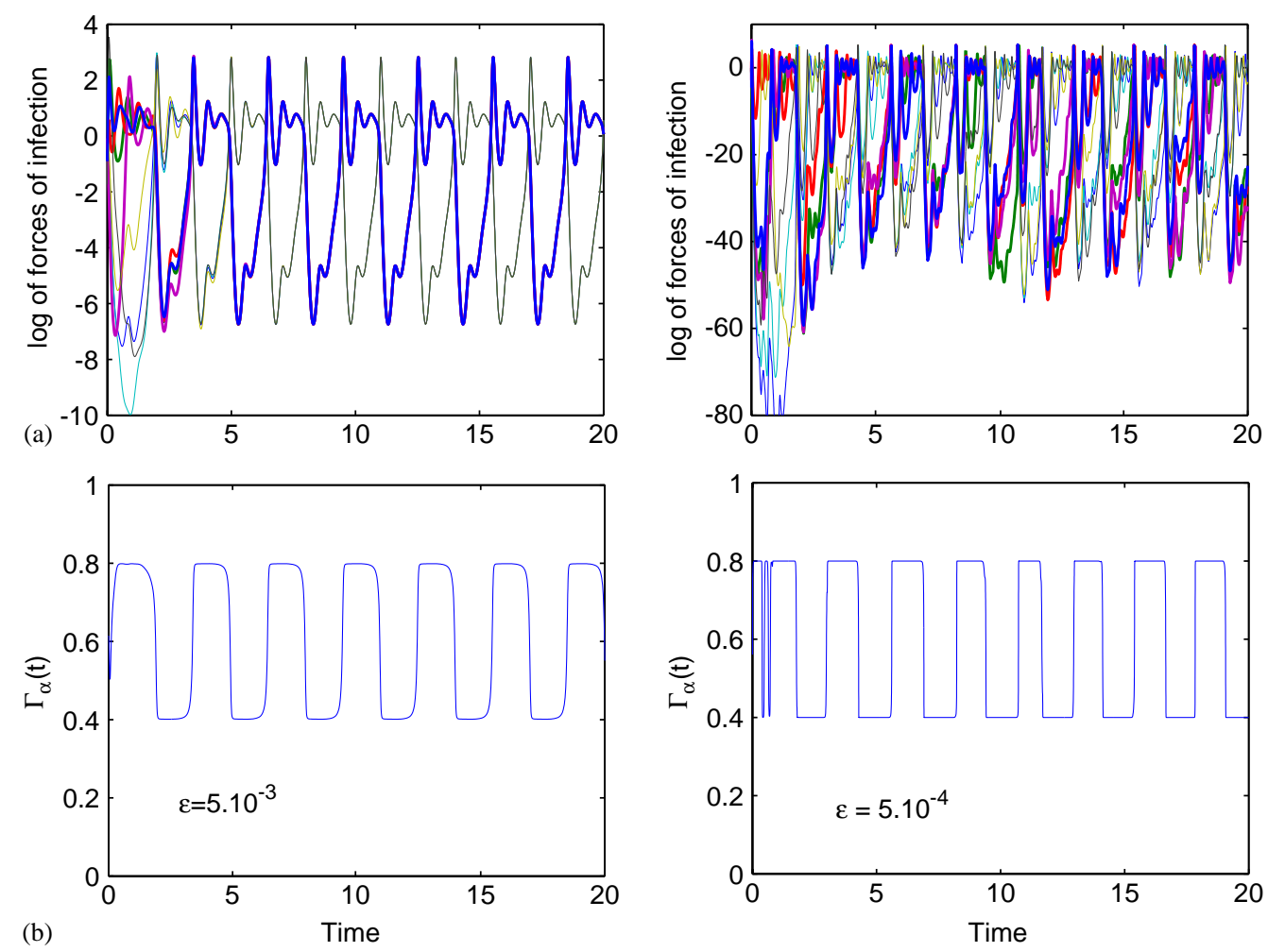

Fig. 4. Dynamics of the logarithm of the forces of infection $Y_{i}(t)\left(\right.$ a) and the effective cross-protection $\Gamma_{\alpha}(t)(\mathrm{b})$ for the "tetrahedric" cluster structure (two clusters with four strains each). The bold lines in (a) are for the forces of infection of the four strains of the cluster $\alpha$. Here $\varepsilon=5 \times 10^{-3}$ (left column) and $5 \times 10^{-4}$ (right column); $\gamma_{1}=0.4$ and $\gamma_{2}=0.8$. Note the remarkable synchronisation of the forces of infection for each cluster for $\varepsilon=5 \times 10^{-3}$ and regularity of the function $\Gamma_{\alpha}(t)$ contrasting to the chaotic behaviour of the forces of infection for $\varepsilon=5 \times 10^{-4}$.

$$
M_{I I}=\left(\begin{array}{llllllll}
1 & 0 & 1 & 0 & 1 & 0 & 1 & 0 \\
0 & 1 & 0 & 1 & 0 & 1 & 0 & 1 \\
1 & 0 & 1 & 0 & 1 & 0 & 1 & 0 \\
0 & 1 & 0 & 1 & 0 & 1 & 0 & 1 \\
1 & 0 & 1 & 0 & 1 & 0 & 1 & 0 \\
0 & 1 & 0 & 1 & 0 & 1 & 0 & 1 \\
1 & 0 & 1 & 0 & 1 & 0 & 1 & 0 \\
0 & 1 & 0 & 1 & 0 & 1 & 0 & 1
\end{array}\right),
$$

respectively. The idea of clustering matrix allows us to define the concepts of cluster structure and type of cluster structure rigorously. The cluster structures are of the same type if their clustering matrices can be transformed one into another by row and column permutations. We also define the effective correlation matrix $R$ with the coefficients (Anishchenko et al., 2002)

$$
R_{i j}=\frac{\left\langle Y_{i}(t), Y_{j}(t)\right\rangle_{T}-\left\langle Y_{i}(t)\right\rangle_{T}\left\langle Y_{j}(t)\right\rangle_{T}}{\sqrt{\left(\left\langle Y_{i}(t)^{2}\right\rangle_{T}-\left\langle Y_{i}(t)\right\rangle_{T}^{2}\right)\left(\left\langle Y_{j}(t)^{2}\right\rangle_{T}-\left\langle Y_{j}(t)\right\rangle_{T}^{2}\right)}} .
$$

Here $\langle Y(t)\rangle_{T}$ is the mean average

$$
\langle Y(t)\rangle_{T}=\frac{1}{T} \int_{\mathscr{I}} Y(\tau) \mathrm{d} \tau
$$

The time interval $\mathscr{I}$ should be sufficiently long and exclude the transient regime. The correlation coefficient $R_{i j}$ is a measure of synchronisation of the time series for the forces of infection; $R_{i j}=1$ when complete synchronization occurs, and $R_{i j}=-1$ when the strains are in antiphase. Naturally, $R_{i i}=1$. For example, for the case shown in Fig. $4, R_{i j}$ is equal to 1 for the strains of the same cluster and to -0.6 otherwise when $\varepsilon=5 \times 10^{-3}$ (left column), and $R_{i j}$ is between 0.75 and 1 for the strains in the same cluster and between -0.45 and -0.19 otherwise when $\varepsilon=5 \times 10^{-4}$ (right column).

It is thereby a fairly straightforward procedure to relate the coefficients $R_{i j}$ to the coefficients of clustering $m_{i j}$. For instance, a threshold function of the form $H(r)=\frac{\exp (\alpha(r-a))}{1+\exp (\alpha(r-a))}$, where $\alpha>0$ is sufficiently large and $0 \leqslant a<1$, can be applied to the elements of the matrix $R$. Fig. 6 illustrates the Euclidean distance between the effective correlation matrix $H(R)$ and the second type clustering 

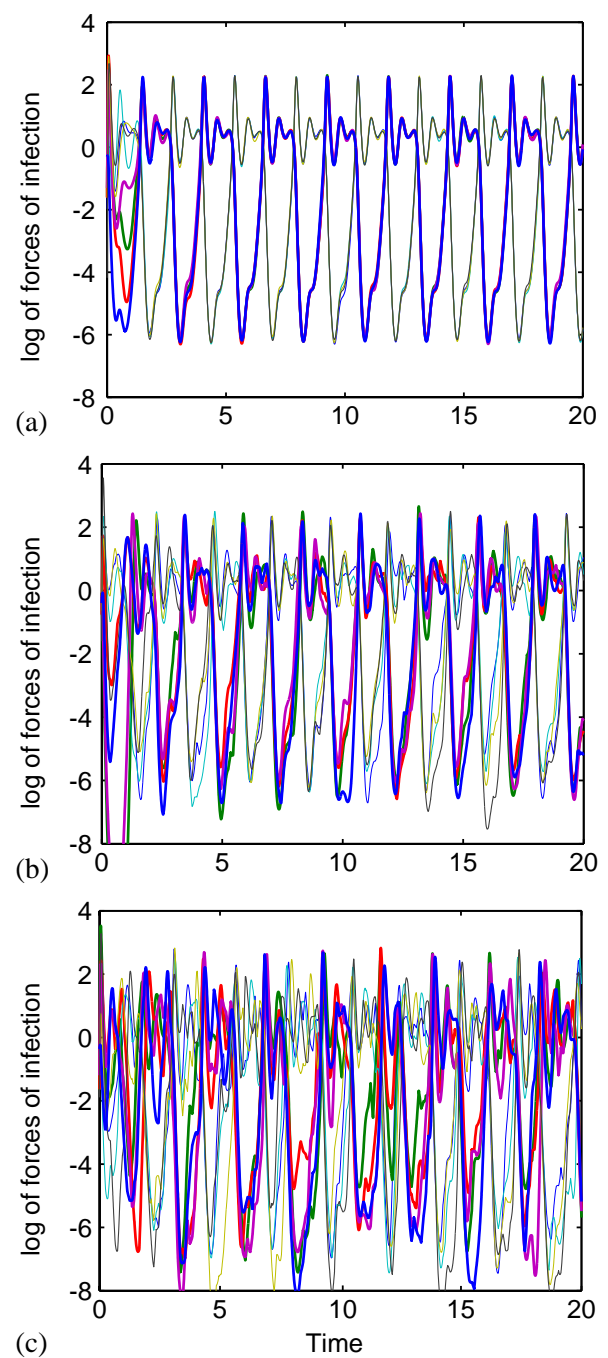

Fig. 5. The logarithm of the forces of infection $Y_{i}(t)$ for the "tetrahedric" cluster structure with stochastic perturbations. Here $1 / \varepsilon=200+\delta(t)$, where $\delta(t)$ is the Gaussian-distributed noise of magnitudes 1 (a), 10 (b) and 20 (c); $r=0.25, \gamma_{1}=0.5$ and $\gamma_{2}=0.8$. The bold lines are for the strains of the first cluster.

matrix $M_{I I}$,

$\Delta=\left\|H(R)-M_{I I}\right\|_{2}=\left(\sum_{i, j}\left(R_{i j}-M_{i j}\right)^{2}\right)^{\frac{1}{2}}$,

for different values of $\gamma_{1}$ and $\gamma_{2}$. (Here $\alpha=20$ and $a=0.7$.) It is easy to see that when $\gamma_{1} \approx \gamma_{2}$, the rapid shift between the two types of clustering occurs.

In some cases, for instance when the system is near an equilibrium state, calculation of the coefficients $R_{i j}$ may be difficult. Then a similarity matrix with the coefficients

$S_{i j}^{2}=\frac{\left\langle\left(Y_{i}(t)-Y_{j}(t)\right)^{2}\right\rangle_{T}}{\sqrt{\left\langle Y_{i}(t)^{2}\right\rangle_{T}\left\langle Y_{j}(t)^{2}\right\rangle_{T}}}$

may be calculated.

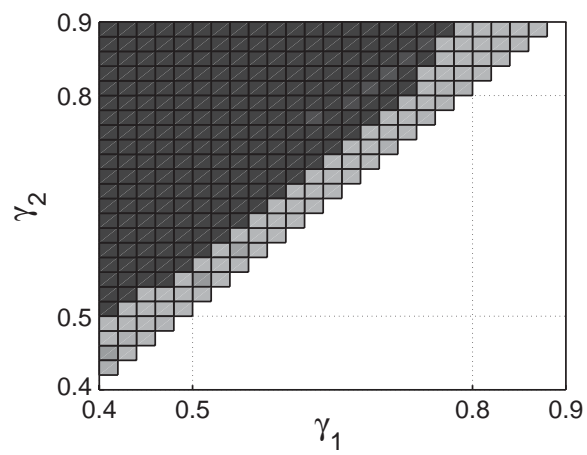

Fig. 6. Euclidean distance $\Delta$ between the effective correlation matrix $H(R)$ and the clustering matrix $M_{I I}$ as a function of $\gamma_{1}$ and $\gamma_{2}$. In the black area $\Delta=1$, that is the matrix $H(R)$ coincides with $M_{I I}$. In the grey area $\Delta=0$; the first type of clustering occurs in this area. The white area corresponds to the biologically unfeasible case $\gamma_{1}>\gamma_{2}$ (the third type of clustering, mentioned in the text, occurs in this area)

New types of clustering may be obtained by breaking the natural constraint $\gamma_{1} \leqslant \gamma_{2}$ or by introducing a non-zero level of cross-protection $\gamma_{0}$ between the discordant strains. For instance, the extremal case $\gamma_{2} \ll \gamma_{1}$ generates a new type of cluster structure: four clusters of two neighbouring strains. Three different cluster structures,

$\left(\begin{array}{llllllll}1 & 1 & 0 & 0 & 0 & 0 & 0 & 0 \\ 1 & 1 & 0 & 0 & 0 & 0 & 0 & 0 \\ 0 & 0 & 1 & 1 & 0 & 0 & 0 & 0 \\ 0 & 0 & 1 & 1 & 0 & 0 & 0 & 0 \\ 0 & 0 & 0 & 0 & 1 & 1 & 0 & 0 \\ 0 & 0 & 0 & 0 & 1 & 1 & 0 & 0 \\ 0 & 0 & 0 & 0 & 0 & 0 & 1 & 1 \\ 0 & 0 & 0 & 0 & 0 & 0 & 1 & 1\end{array}\right),\left(\begin{array}{llllllll}1 & 0 & 0 & 1 & 0 & 0 & 0 & 0 \\ 0 & 1 & 1 & 0 & 0 & 0 & 0 & 0 \\ 0 & 1 & 1 & 0 & 0 & 0 & 0 & 0 \\ 1 & 0 & 0 & 1 & 0 & 0 & 0 & 0 \\ 0 & 0 & 0 & 0 & 1 & 0 & 0 & 1 \\ 0 & 0 & 0 & 0 & 0 & 1 & 1 & 0 \\ 0 & 0 & 0 & 0 & 0 & 1 & 1 & 0 \\ 0 & 0 & 0 & 0 & 1 & 0 & 0 & 1\end{array}\right)$

and $\left(\begin{array}{cccccccc}1 & 0 & 0 & 0 & 0 & 1 & 0 & 0 \\ 0 & 1 & 0 & 0 & 1 & 0 & 0 & 0 \\ 0 & 0 & 1 & 0 & 0 & 0 & 0 & 1 \\ 0 & 0 & 0 & 1 & 0 & 0 & 1 & 0 \\ 0 & 1 & 0 & 0 & 1 & 0 & 0 & 0 \\ 1 & 0 & 0 & 0 & 0 & 1 & 0 & 0 \\ 0 & 0 & 0 & 1 & 0 & 0 & 1 & 0 \\ 0 & 0 & 1 & 0 & 0 & 0 & 0 & 1\end{array}\right)$

are possible for this type. (It is noteworthy that these cluster structures are in the same orbit under the action of cube rotations.)

For a multi-strain system, the dynamics of a single strain is sometimes chaotic ${ }^{4}$ (Gupta et al., 1998). However, under the same conditions which cause

\footnotetext{
${ }^{4}$ Here and through this paper, by the term "chaos" we imply deterministic chaos.
} 
chaotic strain dynamics, clusters usually behave in a surprisingly regular fashion. This regularity is hardly to be expected a priori. To describe the cluster dynamics, for each cluster, e.g. $\alpha$, we define the relative force of infection $\Theta_{\alpha}(t)$ of the cluster as

$\Theta_{\alpha}(t)=\sum_{j \in \alpha} Y_{j} / \sum_{i=1}^{n} Y_{i}$.

Naturally, $0 \leqslant \Theta_{\alpha} \leqslant 1 ; \quad \Theta_{\alpha} \rightarrow 1$ when the cluster $\alpha$ dominates, and it tends to zero when one of the other clusters dominates. Fig. 7 illustrates the dynamics of the cluster $\alpha$ of the eight-strain system: it is easy to see that the evolution of the relative force of infection of the cluster $\Theta_{\alpha}(t)$ is notably regular, while the dynamics of single strains is chaotic. The behaviour of the cluster $\beta$ is qualitatively the same.

The tendency of the strains to self-organise into clusters, and the remarkable regularity of the dynamics of these clusters, contrasting to the chaotic behaviour of a single strain, remains as the number of strains grows. With an increasing number of strains, the number of cluster structures possible for the system grows as well, and new types of cluster structures appear. The 16-strain system, such that for each strain there are four loci with
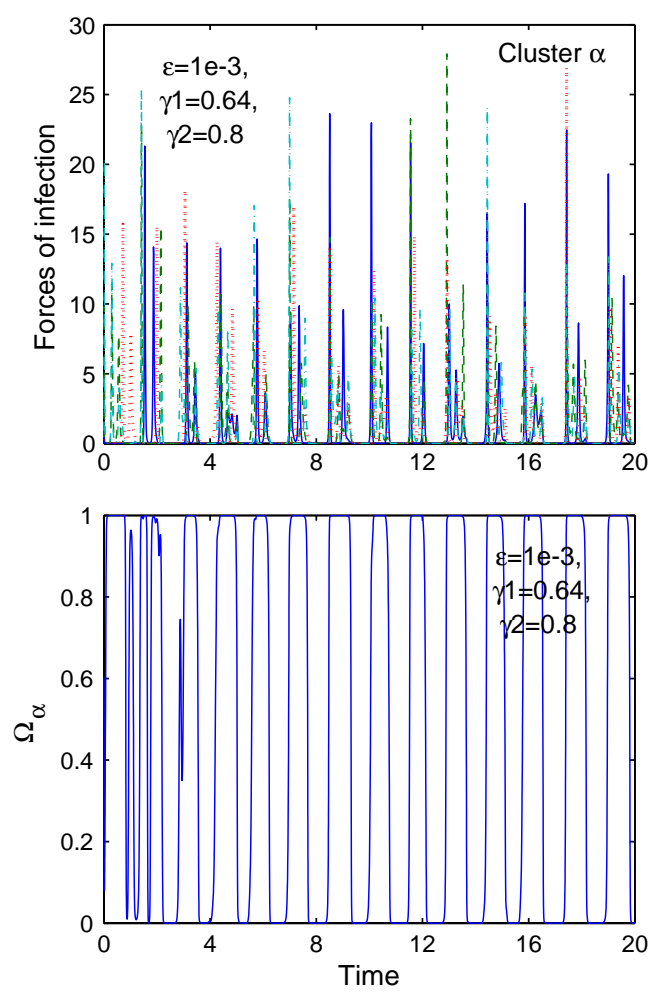

Fig. 7. Dynamics of forces of infection and relative force of infection $\Theta_{\alpha}(t)$ of the cluster $\alpha$ of the eight-strain system (Fig. 2) for the second type of clustering. (Here $\varepsilon=1 \times 10^{-3}, \gamma_{1}=0.64$ and $\gamma_{2}=0.8$.) Note that while the dynamics of each single strain is chaotic, the cluster as a whole behaves remarkably regularly. two alleles possible at each locus, may be visualised as a four-dimensional cube. We assume three levels of crossprotection for this system: $\gamma_{1}$ if the strains share one allele, $\gamma_{2}$ if the strains share two alleles and $\gamma_{3}$ for the strains sharing three alleles (naturally, $\gamma_{1} \leqslant \gamma_{2} \leqslant \gamma_{3}$ ). At least six cluster structures of three different types are possible for this system. Particularly, if $\gamma_{3}, \gamma_{2}$ and $\gamma_{1}$ are approximately equal, a structure of eight clusters with two discordant strains each appears (each cluster corresponds to a main diagonal of the four-dimensional cube; strains of a cluster are the ends of the diagonal). If $\gamma_{3}$ is sufficiently large compared with $\gamma_{1}$ and $\gamma_{2}$, then the system self-organises into two clusters of eight strains each. In this case the strains of a cluster share either no allele at all, or two alleles; there is no cluster with strains sharing one allele in this case. If both $\gamma_{3}$ and $\gamma_{2}$ are large compared with $\gamma_{1}$, then a new stable type of cluster structure appears. In this case eight clusters with two strains each form. A structure of this type differs from the above mentioned structure of the first type (eight clusters with two discordant strains each) as follows: in this case the strains of each cluster share one allele which is at the same locus for every cluster of the structure. That is for this cluster structure, the strains belong to the diagonal of the three-dimensional sides of the fourdimensional cube whereas for the cluster structure of the first type the strains are those on the main diagonal of the four-dimensional cube. Since there are four loci for this system, four different structures of this type are possible.

For this system one may also expect formation of a cluster structure of four clusters with four strains each. However, such a cluster structure was not found for biologically feasible coefficients $\gamma$ (that is for $\gamma_{1} \leqslant \gamma_{2} \leqslant \gamma_{3}$ ). We have been able to generate this cluster structure only for $\gamma_{2}$ larger than $\gamma_{1}$ and $\gamma_{3}$.

Self-organisation of strains into clusters is not a particular feature of the model considered. Computations show that, for an ordered strain system given, the same cluster structures arise for other models, even if the dynamics of these clusters differ. It appears that a cluster structure is intrinsic to an ordered strain system.

For comparison purposes, we considered the models suggested by Gog and Grenfell (2002), and Gog and Swinton (2002). The Gog and Grenfell model is a comparatively simple $S I R$ model composed for $2 n$ classes and purposed to investigate the role of crossimmunity in antigenic drift with a large number of strains. The model equations are

$$
\begin{aligned}
\frac{\mathrm{d} S_{i}}{\mathrm{~d} t} & =\mu-S_{i} \sum_{j=1}^{n} \gamma_{i j} \beta_{j} I_{j}-\mu S_{i}, \\
\frac{\mathrm{d} I_{i}}{\mathrm{~d} t} & =\beta_{i} I_{i} S_{i}-r_{i} I_{i},
\end{aligned}
$$


where $S_{i}$ and $I_{i}$ are, respectively, the fractions of susceptibles and infectives for the $i$ th strain, and $\gamma_{i i}$ is postulated to be equal to one. In contrast, the Gog and Swinton model is a very complex model comprising $n+$ $2^{n}$ classes. If $Z_{J}$ denotes the individuals who are immune to all the strains of the set $J \subset\{1, \ldots, n\}$ (thus they are susceptibles to all the strains which are not in the set $J$ ) then the system equations are:

$$
\begin{aligned}
\dot{I}_{i}= & \beta_{i} I_{i} \sum_{J: i \notin J} Z_{J}-\left(\mu+\sigma_{i}\right) I_{i}, \\
\dot{Z}_{J}= & \sum_{i, K} C(K, J, i) \beta_{i} I_{i} Z_{K}-\sum_{i \notin J} \beta_{i} I_{i} Z_{J} \\
& -\mu Z_{J}+\mu \delta_{J, \emptyset} .
\end{aligned}
$$

The term $C(K, J, i)$ represent the effect of crossimmunity. In fact, it is the rate of transfer from compartment $K$ to compartment $J$ after infection by the strain $i$.

Despite the huge difference in model complexity, both models demonstrate similarities in cluster formation. The behaviour of both these systems is somewhat simpler than that of Gupta's model. Particularly, no alternation of clusters was observed for these models: depending on the system parameters, the phase trajectories of the system converge towards one of the system equilibria with damped oscillations. Nevertheless, the same cluster structures were formed for both of these models. These cluster structures coincide with those for the modified Gupta's model (3), and the values of the cross-protection parameters at which the system shifts from one type of structure to another vary insignificantly from one model to the others. For instance, for the eight-strain system Fig. 2, the type of cluster structure formed depends on the comparative values of $\gamma_{i j}$. As in the case of the system (3), a structure of four clusters with two discordant strains each appears when $\gamma_{1}$ and $\gamma_{2}$ are comparatively close and, as the difference between $\gamma_{2}$ and $\gamma_{1}$ grows, a shift to the structure of the second type (two clusters with four strains each) occurs. However, in contrast to the model (3), no regular oscillation of the clusters was observed: for both types of cluster structures solutions of the models tend to an equilibrium state.

\section{Conclusion}

Strains of a multi-strain infection tend to self-organise into groups, or clusters. For a complex strain system several different types of cluster structures are possible and may arise. Which cluster structure occurs in reality depends mostly on levels of cross-protection and, in some cases, on initial conditions. It is important to note the distinction between the terms "cluster structure" and "type of cluster structure", as several structures of the same type are possible for complex strain systems. Cluster structures which are possible for a strain system do not depend on the particular model used. In fact, the structures are fairly robust to different models. It appears that cluster structures of a particular strain system depend on the structure of the strain space and on levels of cross-protection.

It is not clear why some cluster structures arise while others do not. While clustering in the four-strain system is transparent enough, it is already not so clear why in the eight-strain system the cluster structure of second type (two clusters of four strains) appears. More complex systems, such as the sixteen-strain system, raise even more questions. For instance, it is not clear why no structure of four clusters with four strains each is possible for biologically feasible coefficients of crossprotection. It is a challenge to provide an exhaustive list of type of clustering which may occur for a given set of strain.

One possible interpretation of the phenomenon is that cross-immunity, suppressing some strains, forms negative feedback between the corresponding vertices of the graph (such as in Figs. 1 and 2). This, in turn, induces a positive feedback on other vertices. The phenomenon of self-organisation of elements of an ordered system into clusters does not only occur in epidemiology: for instance, similar examples are observed in neural networks, and we believe that it may occur in other applications. In fact, it appears to be general for coupled dynamical systems. Clustering is a type of self-organisation similar to pattern formation.

The most remarkable feature of the clusters is that they behave remarkably regularly (at least for ordered strain sets), in contrast to the generally chaotic behaviour of isolated strains. Furthermore, a cluster structure, once formed, appears to be exceptionally stable. This stability implies that in many cases we can (and even should) consider the dynamics of a few clusters, instead of the dynamics of multiple separate strains, reducing in this way the system size.

\section{References}

Andreasen, V., Lin, J., Levin, S.A., 1997. The dynamics of cocirculating influenza strains conferring partial cross-immunity. J. Math. Biol. 35, 825-842.

Anishchenko, V.S., Astakhov, V.V., Neiman, A.B., Vadivasova, T.E., Schimansky-Geier, L., 2002. Nonlinear Dynamics of Chaotic and Stochastic Systems. Springer, Berlin.

Cliff, A.D., Haggett, P., Ord, J.K., 1986. Spatial Aspects of Influenza Epidemics. Pion, London.

Dawes, J.H.P., Gog, J.R., 2002. The onset of oscillatory dynamics in models of multiple disease strains. J. Math. Biol. 45, 471-510.

Gog, J.R., Grenfell, B.T., 2002. Dynamics and selection of many-strain pathogens. Proc. Natl Acad. Sci. 99, 17209-17214.

Gog, J.R., Swinton, J., 2002. A status-based approach to multiple strain dynamics. J. Math. Biol. 44, 169-184. 
Gupta, S., Anderson, R.M., 1999. Population structure of pathogens: the role of immune selection. Parasitol. Today 15, 497-501.

Gupta, S., Trenholme, K., Anderson, R.M., Day, K.P., 1994. Antigenic diversity and the transmission dynamics of Plasmodium falciparum. Science 263, 961-963.

Gupta, S., Maiden, M.C.J., Feavers, I.M., Nee, S., May, R.M., Anderson, R.M., 1996. The maintenance of strain structure in populations of recombining infectious agents. Nat. Med. 2, 437-442.

Gupta, S., Ferguson, N., Anderson, R.M., Chaos, 1998. Persistence, and evolution of strain structure in antigenically diverse infectious agents. Science 280, 912-915.

Lin, J., Andreasen, V., Levin, S.A., 1999. Dynamics of influenza A drift: the linear three-strain model. Math. Biosci. 162, 33-51.
Plotkin, J.B., Dushoff, J., Levin, S.A., 2002. Hemagglutinin sequence clusters and the antigenic evolution of influenza A virus. Proc. Natl Acad. Sci. 99, 6263-6268.

Rubin, J., Terman, D., 2000a. Geometric analysis of population rhythms in synaptically coupled neuronal networks. Neural Comput. 12, 597-645.

Rubin, J., Terman, D., 2000b. Analysis of clustered firing patterns in synaptically coupled networks of oscillators. J. Math. Biol. 41, 513-545.

Terman, D., Lee, E., 1997. Partial synchronization in a network of neural oscillators. SIAM J. Appl. Math. 57, 252-293.

Terman, D., Kopell, N., Bose, A., 1998. Dynamics of two mutually coupled slow inhibitory neurons. Physica D 117, 241-275. 